\title{
PROCESOS DE NEGOCIACIÓN DE SIGNIFICADO EN UNA ESCUELA NORMAL MEXICANA
}

\author{
Pedro Antonio Estrada Rodríguez \\ Universidad Pedagógica Nacional, México D.F., México \\ Ruth Mercado Maldonado \\ Centro de Investigación y de Estudios Avanzados del Instituto Politécnico Nacional, México D.F., México
}

\begin{abstract}
RESUMO: Neste trabalho analisamos alguns processos de negociação de significado e desenvolvimento de micropolíticas nas experiências dos docentes e autoridades em uma escola normal mexicana após a reforma à educação normal de 1997. Neste programa de estudos estipula-se que os estudantes realizem os estágios escolares durante o último ano da faculdade. $\mathrm{O}$ estudo foi focado nas atividades que realizaram os docentes de forma prévia aos estágios do pré-serviço com a primeira geração de estudantes do plano de 1997 do Bacharelado em Educação Primária (Ensino Básico). No artigo mostramos como os docentes e autoridades da normal do estudo, transitaram por um processo de negociação de significado e geração de micro-política na sua realização com a proposta curricular nacional e a maneira com a qual foram organizados, sob um esquema que concebemos como comunidade de prática cultivada.
\end{abstract}

PALAVRAS CHAVE: reforma educativa; formação de docentes; comunidade de prática, micropolítica

RESUMEN: En este trabajo, analizamos algunos procesos de negociación de significado y desarrollo de micropolíticas en las experiencias de docentes y directivos en una escuela normal mexicana con la reforma a la educación normal de 1997. En dicho plan de estudios se estipula que los estudiantes realicen prácticas escolares durante el último año de la carrera. El estudio se enfocó en las actividades que realizaron los docentes de manera previa a las prácticas del preservicio con la primera generación de estudiantes del plan de 1997 de la Licenciatura en Educación Primaria. En el artículo mostramos cómo los docentes y el personal directivo de la normal del estudio, transitaron por un proceso de negociación de significado y generación de micropolíticas en su relación con la propuesta curricular nacional y la forma en que se organizaron bajo un esquema que concebimos como comunidad de práctica cultivada.

PALABRAS CLAVE: reforma educativa; formación inicial de docentes; comunidad de práctica, micropolítica.

\section{PROCESSES OF NEGOTIATION OF MEANING \\ IN A MEXICAN PROGRAM OF TEACHER EDUCATION}

ABSTRACT: In this paper, we analyze the experiences of teachers and principals in a Mexican teachers' college following the teacher education reform of 1997. In the aforementioned study program, it is stipulated that students undertake practice in schools during the final year of their degree course. The study focuses on the activities carried out by teachers prior to the pre-service practice with the first generation of students from the 1997 Primary Education degree program. In the paper, we show how the teachers and administrative staff at the teachers' college have adapted by means of a process of negotiation of meaning and the generation of micropolitics in their relationship with the national proposal, and the way in which they are organized as part of a scheme that we conceive as community of practice cultivated.

KEY WORDS: educational reforms; teacher education; community of practice; micropolitic.

En el presente estudio mostramos el desarrollo de una comunidad de práctica cultivada, así como los procesos de negociación de significado y micropolíticas, durante una experiencia vivida por docentes, directivos ${ }^{1}$ y estudiantes de una escuela normal en México. Esta experiencia tuvo lugar en el contexto de la reforma del plan de estudios de 1984 (P84), que fue sustituido por el de 1997 (P97) en la formación inicial de docentes de educación primaria, el cual aún está vigente. ${ }^{2}$ Este plan de estudios incorporó, como parte central del cambio curricular, un periodo de prácticas del preservicio en los dos últimos semestres $\left(7^{\circ}\right.$ y $\left.8^{\circ}\right)$ de la carrera. Los procesos estudiados se relacionan con la puesta en práctica del preservicio de los estudiantes de la normal del estudio en las escuelas primarias, considerando la experiencia de la primera generación del P97.

En los últimos años, las investigaciones sobre la formación en diferentes profesiones han puesto su mirada en los procesos de la práctica (Bullogh, Jr.,1997; Coll, 1990; Fullan, 2002; Hargreaves, 2001; Schön, 1987;). De acuerdo con esos autores la práctica se ejecuta al interior de comunidades de práctica (Lave y Wenger, 1991) o 
en las llamadas comunidades de aprendizaje (Putnam y Borko, 1997).

En el modelo expuesto por Wenger (1998), la práctica pasa por el proceso de negociación de significado, noción que es una de las bases de nuestro trabajo. Por otra parte, autores como Ball (1987) y Blase, (1997) explican de manera precisa, la forma en que los docentes y directivos de las escuelas proyectan acciones o micropolíticas para subsistir o sobresalir en sus escuelas. Estas tendencias conceptuales forman parte de una perspectiva teórica que algunos han denominado enfoque socio-cultural. Cole (1996), expone un recorrido histórico de ese enfoque ubicando a Vygotski como un antecedente común.

Desde ese enfoque socio-cultural, en nuestro trabajo planteamos la existencia de procesos de mediación o negociación de significado entre los docentes de la normal del estudio por un lado y los planteamientos oficiales del P97 por otro, con la primera generación de estudiantes que harían sus prácticas con ese plan. En esa negociación de significado destacamos de manera central las experiencias, creencias y expectativas de aquellos docentes que protagonizaron la organización del preservicio de los estudiantes del $7^{\circ}$ y $8^{\circ}$ semestres de la licenciatura en educación primaria en la normal del estudio.

También destacamos en este trabajo los procesos a través de los cuales los docentes se organizaron para resolver las situaciones cotidianas que les planteo la reforma del plan de estudios en relación con las prácticas del preservicio de los estudiantes. Al respecto, encontramos que la dinámica desarrollada por los docentes correspondió a lo que algunos autores como Wenger, McDermott \& Snyder (2002), denominan comunidad de práctica cultivada.

En esa experiencia los docentes confrontaron sus ideas, construyeron acuerdos, tomaron decisiones y generaron micropolíticas desde las cuales replantearon las acciones que proponía el P97 para las prácticas escolares de los estudiantes de la normal.

Presentamos el trabajo en cuatro partes; primeramente exponemos la perspectiva conceptual desde la cual analizamos las situaciones registradas en la investigación; en la segunda parte describimos la normal del estudio; en tercer lugar, analizamos el desarrollo de una comunidad de práctica cultivada por los profesores de la normal. Finalmente, exponemos dos ejemplos sobre los procesos de negociación de significado y micropolítica en los que se involucraron los docentes en torno a las prácticas del preservicio de los estudiantes con el P97.

Una Comunidad de Práctica, para Organizar el Preservicio de los Estudiantes de Normal

Partiendo del enfoque socio-cultural, algunos trabajos como los de Bullough Jr. (1997), Chainklin y Lave (1996), Lave y Wenger (1991), Schön (1987), Salomon (1993), Rogoff (1990), Putnam y Borko (1997), así como Wenger (1998), entre otros, explican los procesos de construcción y apropiación de los saberes culturales y profesionales en contextos sociales específicos.

En esa perspectiva se plantea que la formación profesional se genera en la participación y que puede ubicarse en las comunidades de práctica, por lo tanto el aprendizaje de la profesión u oficio se construye en las situaciones cotidianas, en las acciones mediante las cuales se resuelven o se intenta resolver los problemas de la práctica profesional.

Las comunidades de práctica no son un acontecimiento nuevo, según lo señalan Wenger, McDermott \& Snyder (2002, p. 5), "ya que existían en el tiempo de las cavernas, cuando nos reuníamos alrededor del fuego a discutir estrategias para la cacería, la pesca y para saber cuáles eran las raíces comestibles". Sin embargo, estos autores precisan que la conceptualización de esos procesos es un acontecimiento reciente.

Para los precursores de estos conceptos, las comunidades de práctica son grupos de personas que comparten intereses, problemas, o una pasión acerca de un tema. Es decir, "los integrantes de las comunidades se reúnen para interactuar y compartir información valiosa y porque entre ellos surgen nuevas ideas, información, consejos y también porque se ayudan a resolver problemas profesionales" (Wenger, McDermott \& Snyder, 2002, p. 4).

Las aportaciones teóricas de esos autores conciben como cuestión central el contexto cultural y social respecto al aprendizaje de los conocimientos prácticos profesionales. Desde esa postura, el punto central para estudiar la producción de conocimientos no es el individuo, sino el proceso que media entre lo cultural y lo individual, es decir la participación de los sujetos en los procesos sociales.

Otro concepto subsidiario de la perspectiva teórica antes mencionada y que orienta nuestro análisis, es el de negociación de significado. Este concepto se encuentra integrado al de comunidad de práctica (Wenger, 1998, 77). La negociación de significado tiene lugar en el proceso de interacción de las personas con el mundo y se relaciona con la forma en que éstas participan en las comunidades de práctica. Según este autor, la negociación de significado es participación.

En ese sentido, entendemos que en los procesos de negociación de significado las personas, interactúan y se aproximan a otras a partir de sus saberes y en esa aproximación reconocen el saber del otro. En el proceso de negociación existe un intercambio de significados a partir de los cuales los interlocutores se entienden, a pesar de que sus referencias y experiencias sean diferentes. Wenger señala que la negociación de significado tiene lugar en las situaciones de la práctica cotidiana o profesional; considerando que para los miembros de una comunidad de práctica, los procesos y las cosas tienen un sentido ligado a la cultura (Wenger,1998).

La micropolítica, según Blase (1997), remite al uso del poder formal e informal por parte de los individuos y 
los grupos para alcanzar sus objetivos dentro de las organizaciones. Las acciones políticas son en gran medida el resultado de las diferencias que se perciben entre los individuos y los grupos, unido a la motivación de emplear el poder para influir y/o protegerse. "Tanto las acciones y los procesos cooperativos como los conflictos forman parte del reino de la micropolítica" (Blase, 1997, p. 256). Hoyle describe a "la micropolítica como las estrategias con las cuales los individuos y grupos que se hallan en contextos educativos tratan de usar sus recursos de poder e influencia a fin de promover sus intereses" (Ball, 1987, p. 34-35).

Para precisar nuestro análisis hemos vinculado la noción de micropolítica con la de negociación de significado, enfocando las acciones emprendidas por los protagonistas de nuestro estudio al llevar a la práctica sus ideas y planificaciones. En la literatura producida en este campo nos encontramos con estudios como los de Duverger (1972), Hoyle (1982), Ball (1987), Blase (1997), Kelchtermas y Ballet (2002), quienes han explicado y conceptualizado el comportamiento de los directores y maestros en el trabajo de gestión y planificación en los centros escolares.

Esos análisis se orientan hacia una perspectiva individual de los docentes del estudio destacando sus intereses, inquietudes, incertidumbres, así como las situaciones y condiciones materiales en las que desarrollan sus actividades cotidianas. También promueven la comprensión de las formas en las que los docentes organizan sus acciones y la influencia que éstos tienen con sus colegas, grupos políticos e institución.

Desde esta línea de interpretación se ha generado una mirada profunda que analiza la forma en que los maestros y directores construyen un estilo personal de actuación frente al mundo que los rodea. Las determinaciones que ellos toman en las tareas de planificar y desarrollar su trabajo, han sido denominadas micropolíticas.

En México la literatura existente sobre el desarrollo de las prácticas escolares del preservicio en la formación inicial de docentes es muy escasa, existen trabajos como los de Czarny (1999) y Mercado (2000) que reportan aspectos relacionados con la experiencia de los primeros semestres del P97. Estos trabajos se realizaron antes de que los estudiantes de la primera generación de ese plan asistieran a las prácticas del preservicio.

En otros países, donde las prácticas de preservicio se implementaron antes que en México, se han realizado trabajos que analizan las situaciones y dificultades que enfrentan los estudiantes de magisterio durante las prácticas escolares (Gal, 2006). Otros trabajos analizan la relación entre los cursos de la escuela formadora de docentes y los problemas de los estudiantes en las primarias durante las prácticas escolares (Kelchtermans y Ballet, 2002; Wubbels, Korthagen y Brekelmans, 1997;). Algunos investigadores han experimentado modelos alternativos para el desarrollo de las prácticas del preservicio y estudiado sus efectos (Jay \& Johnson, 2002; Taitel, 1997; Veenman, Benthum, Bootsma \& Van Dieren, 2002).

Los trabajos que encontramos más relacionados con el nuestro, fueron los de Sim (2005) en Australia y el de Wilson (2006) en EUA. El primero se refiere a la construcción de un modelo para sistematizar las actividades de observación y reflexión de las prácticas, mediante la noción de comunidad de práctica. El trabajo de Wilson analiza los problemas que se presentan entre los supervisores de la formación inicial, los tutores de primaria y los estudiantes.

Cabe señalar que en los programas de formación inicial, en la mayoría de las universidades europeas, norteamericanas y latinoamericanas, así como en las normales de México, las prácticas escolares del preservicio se desarrollan con la participación de tres figuras centrales que son el asesor (maestro de la normal que supervisa las prácticas, coordina el Seminario de análisis del trabajo docente y dirige la elaboración del documento de titulación), el tutor (profesor de educación primaria que está a cargo del estudiante) y el estudiante que realiza las prácticas de enseñanza en las primarias (SEP, 2002, p. 17). Estas figuras constituyen lo que Wilson (2006), en EUA llama la "tríada tradicional.".

\section{Ubicación Contextual del Estudio}

Como parte de las reformas educativas en la formación inicial de docentes que se propusieron varios países, a partir de las recomendaciones emanadas de la XXXV y la XLV Conferencias Internacionales sobre Educación de la Organización de las Naciones Unidas para la Educación, la Ciencia y la Cultura (UNESCO) (SEP, 1997c), en México se estableció la carrera de profesor de educación primaria con grado de licenciatura en 1984. Posteriormente, en 1997, de acuerdo con el Programa de Transformación y Fortalecimiento Académico de las Escuelas Normales (SEP, 1997,b) ${ }^{3}$ se modificó el P84, el cual fue sustituido por el $\mathrm{P} 97^{4}$.

Algunos estudios sobre la implantación del P97 señalan que el diseño de ese plan, puso énfasis en el desarrollo de un enfoque orientado a la enseñanza, a diferencia del P84 que apuntaba hacia la formación de docentes-investigadores (Mercado, 1994; Czarny, 1999). En otro trabajo se señala que "las actividades en las materias pedagógicas se orientaban al entrenamiento en metodologías de observación, de tal manera que el estudiante se acercaba a la escuela no como aprendiz de maestro, sino supuestamente como investigador en ciernes" (Mercado, 2000, p. 30).

Bajo la nueva perspectiva de la formación inicial de docentes, en el mapa curricular del P97 se incorporó una 
línea de aproximación a la práctica escolar a lo largo de todo el plan. En ella, los estudiantes debían practicar en las primarias en tres momentos de la carrera; primero como observadores del grupo y de las actividades escolares, después como ayudantes del profesor tutor y finalmente, en el $7^{\circ}$ y $8^{\circ}$ semestres durante el preservicio, haciéndose cargo del grupo bajo la tutoría del profesor responsable (SEP, 1997a, 2002).

En esos dos últimos semestres los estudiantes desarrollan tres actividades centrales que consisten en a) asistir a las prácticas escolares a una escuela primaria bajo la responsabilidad de un profesor de primaria al que se denomina tutor $b$ ) cursar dos seminarios relacionados con las prácticas en la escuela normal (Seminario de trabajo docente y Seminario de análisis del trabajo docente) con un profesor de la normal o asesor y, c) elaborar el documento para la titulación.

La escuela normal en la que ubicamos el estudio, se localiza en el centro de la República Mexicana. La escuela constaba de tres planteles que llamaremos: Unidad Central, ubicada en la capital del estado y Unidades Externas 1 y 2 que se encontraban en otras poblaciones. El estudio se hizo en la Unidad Central donde funcionaban las licenciaturas de Educación Primaria, Educación Preescolar, Educación Física, Educación Especial y Educación Media; nosotros trabajamos con la primera de ellas.

Las prácticas escolares del preservicio de la primera generación de estudiantes de la Licenciatura en Educación Primaria de la normal del estudio, iniciaron en agosto de 2000 y terminaron en junio de 2001, de acuerdo con el calendario de la Subsecretaría de Educación Básica y Nor$\mathrm{mal}^{5}$. Esta fue la primera experiencia de prácticas intensivas de ese tipo, en la normal desde que inició el P97, ya que bajo el esquema del P84 las prácticas se realizaban por períodos cortos de una semana o dos en cada uno de los ocho semestres.

Metodológicamente la investigación se desarrolló bajo una perspectiva etnográfica (Geertz, 1973; Rockwell 1980; Rockwell y Mercado 1986), desde la cual no se trata sólo de describir los hechos y los discursos textuales, sino de encontrar el sentido que éstos tienen en un contexto cultural y en una situación determinados. Este enfoque se caracteriza por "cierto tipo de esfuerzo intelectual: una especulación elaborada en términos de descripción densa" como señala Ryle, citado por Geertz (1973, p. 21).

Considerando esa perspectiva, aunada a la de Woods (1986) y Erickson (1989), realizamos observaciones de varias sesiones de trabajo del personal docente y directivo de la escuela normal. El análisis que aquí presentamos se basa en esa información.

Desde esta orientación, los acontecimientos registrados ${ }^{6}$ fueron organizados e interpretados considerando que las escuelas tienen sus propias tradiciones, normas, creencias y saberes comunes, es decir constituyen una cultura (Viñao, 2001) y desde la visión de Wenger (1998) conforman comunidades de práctica.

Comunidad de Práctica Cultivada y Micropolíticas en la Organización del Preservicio

La puesta en operación del actual plan de formación inicial en México, iniciado en 1997, presentó un desafío para el personal docente de las normales, pues implicó un giro importante en la vida cotidiana de estas instituciones, como lo señalan los estudios de Czarny y Mercado ya mencionados. Por un lado, la orientación conceptual del P97 desplazó a las perspectivas disciplinarias para la formación inicial en nuestro país, presentes en el P84 (Mercado, 1994). En su lugar apareció un currículo diseñado desde una posición epistemológica diferente, con un mayor espacio a lo largo del plan para las prácticas escolares que culminarían, por primera vez, con un año completo de los estudiantes en las primarias.

Ubicados en ese contexto, destacamos la constitución de una comunidad de práctica cultivada constituida en aquellos momentos por el personal docente y directivo de la normal frente a la propuesta para el preservicio planteada por el P97. Esta comunidad de práctica desarrolló algunas de las acciones que sus integrantes consideraron como las más urgentes y necesarias previas a las prácticas escolares.

Cabe señalar que en la época del estudio existían problemas internos en la normal, debidos a las confrontaciones de dos grupos políticos, ambos de maestros y directivos. Sin embargo, para organizar las prácticas del preservicio de los estudiantes con el nuevo plan, los docentes lograron llegar a ciertos acuerdos organizativos. Como parte de ellos, conformaron una comisión que propusiera un proyecto para operar, desde la normal, las prácticas de los estudiantes, incluyendo las gestiones necesarias para que éstas se llevaran a cabo en las primarias. Dicha organización, que en adelante llamaremos "la comisión", fue formada por los docentes con más experiencia y trayectoria de participación académica en la escuela y funcionó bajo la estructura y dinámica de lo que conocemos teóricamente como una comunidad de práctica cultivada.

La comisión quedó constituida con representantes docentes (que no tenían cargos académico-administrativos) de la Licenciatura en Educación Primaria, Licenciatura en Educación Preescolar, Licenciatura en Educación Física, Licenciatura en Educación Media, Licenciatura en Educación Especial, Departamento de Titulación, Departamento de Servicio Social, Departamento de Investigación, Departamento de Difusión Cultural, Departamento de Superación Profesional y Subdirección Académica (todos ellos de la Unidad Central). También había un docente representante de la Unidad Externa 1 y un representante de la Unidad Externa 2. Eventualmente se integraron a la comisión el Director General de la 
Escuela, más los directivos de las licenciaturas y los departamentos señalados.

Durante su gestión, la comisión realizó reuniones de diferente índole; internas o cerradas, sesiones especiales y algunas plenarias para las cuales decidían quiénes debían participar. A las internas solamente asistían los integrantes de la comisión; a las especiales se invitaba a otras personas, como los representantes sindicales y los directivos de algunos departamentos como el de Titulación, Servicio social e Investigaciones educativas, entre otros. A las reuniones plenarias asistían también los directivos de todos los departamentos y licenciaturas de la escuela, así como el Director General. Estas últimas se realizaron cuando la comisión ya tenía propuestas específicas y consideraba importante la presencia del pleno para que se tomaran las decisiones necesarias.

En las primeras reuniones (marzo de 2000, seis meses antes de que se iniciaran las prácticas) los integrantes de la comisión hicieron un análisis de la situación en la que se encontraba la normal en esos momentos. Para ello, consideraron fundamentalmente las horas de docencia y los profesores con que se contaba, comparando esto con los recursos, que según ellos, requerían para cubrir todas las tareas que ahora les demandaba el P97 durante el $7^{\circ}$ y el $8^{\circ}$ semestres.

Sumado a lo anterior, los docentes y el personal administrativo manifestaron en las reuniones una gran preocupación por la falta de información oficial oportuna, sobre las actividades que deberían desarrollar para realizar las prácticas escolares con el nuevo plan.

Otro aspecto que también discutió la comisión en las reuniones fue la necesidad, así expresada por ellos, de diseñar y promover seminarios y talleres de actualización, ya que argumentaban tener dudas sobre el enfoque teórico y las formas para operar las actividades académicas del P97, principalmente en cuanto a las prácticas del preservicio. En las reuniones de la comisión, los docentes manifestaron desconocer las nuevas funciones de los asesores, los tutores y principalmente, cómo trabajarían los estudiantes en las primarias.

La comisión también promovió la formalización de convenios entre las instituciones involucradas en las prácticas del preservicio, como por ejemplo entre la Dirección de Educación Básica en el Estado y la Escuela Normal. Planteaban que esos convenios eran muy importantes, ya que los estudiantes estarían en las primarias por un año y se requería contar con criterios que normaran esas estancias. Argumentaban esto, porque la normal y las primarias pertenecían a subsistemas educativos diferentes por lo cual se regían bajo distintas normas administrativas.

Los docentes de la comisión también gestionaron otros convenios con la representación laboral de los académicos de la normal y con el Sindicato Nacional de
Trabajadores de la Educación (SNTE) ${ }^{7}$ Señalaban que esto era necesario para que los profesores de las primarias asumieran el papel de tutores y para que los docentes de la normal funcionaran como asesores de los estudiantes en las primarias, según lo estipulaba el P97.

Asimismo, la comisión elaboró una propuesta para modificar las funciones del Departamento de Servicio Social (DSS) y del Departamento de Titulación de la normal, ya que éstos cambiarían sus funciones con el nuevo enfoque de las prácticas del preservicio. El P97 proponía que los estudiantes validarían su servicio social (ss) con las mismas prácticas escolares; ya no se requería entonces que el DSS hiciera el seguimiento y la evaluación de las actividades del ss. En cuanto a la titulación, el P97 ya no permitía, como el P84, la elaboración de tesis, tesinas, informes de prácticas, ni trabajos en equipos de dos a ocho estudiantes por documento. Con el P97 la titulación se haría mediante un ensayo basado en las experiencias de enseñanza durante el año de preservicio en las primarias realizado individualmente (SEP, 2000a).

Por otro lado, la comisión elaboró un proyecto de capacitación para los docentes asesores y otro para los tutores, que les diera a conocer el nuevo enfoque pedagógico de la formación inicial y la función de las prácticas escolares.

De acuerdo con nuestro análisis encontramos que la comisión se encontraba inmersa en una dimensión histórica-cultural en la que existían tradiciones, costumbres, normas, así como prácticas profesionales comunes y repertorios para la solución de problemas, tales como la organización y supervisión de los periodos de prácticas escolares que realizaban los profesores de la normal orientados por el anterior P84.

La forma en que se constituyó y funcionó la comisión, así como la experiencia profesional de quienes la integraron, fueron similares a lo que Wenger, McDermott \& Snyder (2002, 49-64), llaman comunidad de práctica cultivada.

Una de las características de esas comunidades, es que surgen para resolver problemas que no han sido resueltos con anterioridad y que representan un reto para las instituciones o empresas. Frente a ello, los interesados promueven la organización de una comunidad de personas experimentadas en la actividad, tema o problema por resolver. Los promotores seleccionan a personas que reúnan ciertas características como dominio del lenguaje específico del oficio o profesión y conocimiento de las formas tradicionales de resolver los problemas. "Las comunidades de práctica cultivadas generan conocimientos o estrategias que los profesionales necesitan” (Wenger, McDermott \& Snyder, 2002, p. 5).

En el caso que aquí presentamos, la comisión, como comunidad de práctica, funcionó con cierta autonomía respecto al personal directivo de la escuela 
normal, y de las autoridades educativas estatales, ya que la comisión tenía autorización de sus jefes inmediatos para tomar decisiones. Además, todos los integrantes de la comisión eran docentes en la normal, es decir, participaban de una práctica profesional común y por tanto compartían campo de trabajo, lenguaje profesional y repertorios de la práctica profesional.

Para nuestro análisis, siguiendo a Wenger (1998, p. 290), el ejemplo de la comisión muestra cómo "las comunidades de práctica tienen su propia empresa" y expectativas particulares a partir de las cuales desarrollan sus acciones y sus estrategias. Aunque estas comunidades hayan surgido como una determinación institucional, su visión del mundo y su forma de resolver las situaciones particulares son propias de la comunidad.

"Muchas comunidades de práctica surgen en el proceso de dar existencia a un diseño institucional; incluso pueden deber su existencia al contexto institucional en el que surgen. Sin embargo, no es el mandato lo que produce la práctica, sino la comunidad" (Wenger, 1998, p. 290).

En la normal del estudio, la empresa de la comisión fue organizar las acciones de toda la institución y de otras involucradas para que los alumnos de $7^{\circ}$ y $8^{\circ}$ semestres practicaran en las primarias. A ese respecto, es importante subrayar que la comunidad de práctica que analizamos no fue producto de un diseño de expertos, sino que surgió de manera emergente, o espontánea.

Para resolver los problemas planteados por la reforma del P97 y específicamente por el nuevo enfoque de las prácticas escolares, los docentes de la normal del estudio partieron de los saberes (Mercado, 2002), que habían generado en sus experiencias con los planes de estudio anteriores al P97 y de sus expectativas sobre cómo deberían ser esas prácticas y la relación entre la normal y las escuelas primarias.

Después de seis meses de intenso trabajo, la comisión logró elaborar y gestionar, convenios con las instituciones relacionadas con las prácticas escolares y con ello contribuyó a preparar las condiciones para que los estudiantes de la normal asistieran por un año a las escuelas primarias.

La Comunidad de Práctica y dos Procesos de Micropolítica sobre el Preservicio

En esta sección del trabajo presentamos dos ejemplos sobre la participación de la comunidad de práctica en procesos de negociación de significado y micropolítica en torno al preservicio de los estudiantes con el P97 en la normal del estudio.

Al involucrarse como comunidad de práctica en la empresa de organizar y desarrollar las prácticas del preservicio de los estudiantes, los profesores protagonizaron procesos de micropolítica frente al nuevo plan de estudios modificando algunos de sus aspectos mediante las acciones que ellos consideraron idóneas. A continuación mostramos dos ejemplos de esos procesos

En el primer ejemplo describimos las distintas perspectivas que tenían los integrantes de la comisión sobre el servicio social de los estudiantes y cómo éstas a su vez diferían de los señalamientos contenidos en el P97 a ese respecto. En el segundo ejemplo nos referimos a la propuesta de la comisión para orientar el trabajo de los estudiantes en las primarias y a las diferencias que había entre ésta y la que planteaba el P97.

Sobre el Servicio Social de los Estudiantes en las Prácticas del Preservicio

En esta parte del texto analizamos la forma en que los docentes de la comisión desarrollaron un proceso de negociación de significado y micropolítica acerca del servicio social (ss) de los estudiantes de la normal en las primarias frente a la propuesta nacional del P97 sobre ese tema.

Los integrantes de la comisión plantearon que el ss debería realizarse por los estudiantes además de las prácticas escolares. En cambio, el P97 indicaba que los estudiantes normalistas podrían validar el ss con las mismas prácticas escolares, sin necesidad de realizar otras actividades (SEP, 2000a, p. 57). Sin embargo, algunos docentes propusieron que los estudiantes realizaran tareas adicionales para cumplir con ese servicio.

En las discusiones que tuvieron sobre el tema, los profesores debatieron si los estudiantes deberían realizar sólo las prácticas de enseñanza entendidas a la vez como ss, o si debían realizar alguna otra actividad y cuál tendría que ser. Las posiciones de los docentes a ese respecto presentaron tres vertientes que, aunque parecidas, tenían ciertas diferencias.

La primera posición fue expresada por docentes que propusieron un ss comunitario, semejante al que hicieron los estudiantes con el anterior P84. En ese plan, el ss consistía en mejorar aspectos físicos de las escuelas primarias y aulas donde practicaban los estudiantes; en elaborar material didáctico y, en algunos casos, organizar actividades culturales con los padres de familia o con la población donde se ubicaba la escuela. Así lo señaló uno de esos docentes cuando se discutió el tema:

Juvencio: Es que por cuestiones de normatividad los estudiantes deben desarrollar ambas funciones [la práctica escolar y el servicio social]. No podemos evadir la normatividad de la institución sobre el servicio social. Por eso existe un departamento de servicio social, y eso quiere decir que el servicio social debe formar parte del trabajo docente. Eso hay que dejarlo claro entre los alumnos, sobre todo por cuestiones de normatividad... pues eso es una determinación institucional. Por lo tanto los estudiantes, además de la práctica docente, deben hacer un servicio social. A1/RCR/22-03-00,5.

En los argumentos del maestro existe, al parecer, una preocupación para que el servicio social continuara 
bajo la organización existente hasta ese momento en la normal, pues el reglamento interno del ss vigente en el momento del estudio, indicaba que los estudiantes (en el P84), hacían actividades adicionales a las prácticas de enseñanza. En ese sentido, la normatividad establecida hasta ese momento para el ss ya no tenía cabida con el P97, lo cual aparentemente dejaba sin funciones al Departamento de Servicio Social aunque, por otro lado, no habíaindicaciones oficiales al respecto.

Una segunda posición de los docentes de la comisión sobre el ss fue la adoptada por los maestros que señalaban que éste debía dirigirse a la comunidad, lo cual era inherente al "ser maestro". Estos docentes propusieron actividades como la formación de grupos de alfabetización, grupos artísticos en la comunidad, actividades culturales, escuela para padres y organización de viajes de estudio. Así lo argumentó uno de los maestros:

Germán: Es que ése es el trabajo del profesor, no sólo esto de dar clases. Vincular a la escuela con la comunidad es parte del trabajo del docente y ésa es la función del profesor... Por eso decía [que lo del servicio social] no debe ser una tarea opcional sino obligatoria, ya que es parte del trabajo docente. Al/ $R C R / 22-03-00,5)$.

Para este maestro, servir a la comunidad es parte del ser docente y debe agregarse, como servicio social, a las tareas de "dar clases."

En la tercera posición sobre cómo debería ser el ss de los estudiantes los docentes manifestaron que el ss debería realizarse como un "pago" de los estudiantes a la sociedad por haber recibido su formación profesional. Esta posición tuvo ciertas coincidencias con la segunda, aunque los fundamentos son diferentes. Uno de los maestros lo expresó así:

Benito: ... El servicio social, según establece el programa rector de esta licenciatura, debe de ser frente al grupo y durante los dos últimos semestres. Pero las actividades de servicio social no deben de confundirse con la práctica profesional, debe ser algo independiente [elaborando material didáctico para los alumnos, desarrollando proyectos de nivelación académica, entre otros] Yo recuerdo bien que la filosofía del servicio social es devolver un poco a la sociedad, lo que invierte a través de sus impuestos, para que los estudiantes lleven a cabo una formación profesional. VI/RT/ 11-05-00,17

Como estos maestros argumentaban el pago a la sociedad, proponían que el ss consistiera en hacer algunas labores en el aula de la primaria, pero de manera diferenciada a las prácticas escolares marcadas en el programa. Sugirieron que los estudiantes hicieran trabajos como elaborar material didáctico, apoyar a los alumnos con problemas de aprovechamiento escolar y desarrollar proyectos alternativos para la enseñanza.
Durante el proceso de análisis colectivo respecto al ss, los integrantes de la comisión entraron en un proceso de negociación de significado con respecto al P97. Por un lado, hicieron públicas y compararon sus diferentes ideas sobre el significado que debía tener el ss de los estudiantes. Al hacerlo, se distanciaban también del sentido con cual el P97 proponía ese servicio.

Como producto de esa negociación de significado, los integrantes de la comisión llegaron a establecer acuerdos respecto a cómo deberían definir las tareas del ss. Se definió que los estudiantes debían hacer un ss que beneficiara a la sociedad y que las actividades fueran diferentes a las prácticas de enseñanza, aunque vinculadas con ellas. Para cumplir con esos requisitos debían elaborar un proyecto que no estaba previsto en el P97.

De esa manera, las decisiones tomadas por los docentes de la comisión acerca del ss resultaron distintas a las recomendaciones de la SEP en el P97 que señalaban que sólo con las prácticas en los grupos, sin agregar otras actividades, los estudiantes validarían el servicio social (SEP, 2000a, p. 57).

Concluyendo esta parte del trabajo, podemos decir que las creencias y experiencias de los docentes de la comisión, así como sus expectativas y formas de interpretar el mundo, fueron algunos de los elementos desde los cuales confirieron sentidos a sus propuestas y a las de los otros en un contexto social determinado. Esas perspectivas, entraron en acción desarrollándose un proceso de negociación de significado frente a la propuesta nacional del P97, que los profesores no retomaron plenamente, pero la tuvieron como un punto de referencia durante todo el proceso.

\section{Acerca de la Orientación Pedagógica de las Prácticas Escolares de los Estudiante}

En esta sección presentamos un segundo proceso de micropolítica desarrollado por la comunidad de práctica que organizó el preservicio de los estudiantes de la normal del estudio. Como en el ejemplo anterior, concebimos el desarrollo de este proceso, como una manera particular de objetivación de la negociación de significado que tuvo lugar en el contexto del estudio respecto a los planteamientos del P97.

El proceso de micropolítica que aquí mostramos, se refiere a las características que tendrían las acciones, sobre todo pedagógicas, que orientarían las prácticas del preservicio de los estudiantes en las primarias durante los últimos semestres $\left(7^{\circ}\right.$ y $\left.8^{\circ}\right)$ en la normal. Como se verá en la descripción, los integrantes de la comisión y las recomendaciones del P97 planteaban diferentes perspectivas al respecto.

Esta parte del análisis se basa en varios fragmentos de una reunión y sus antecedentes, a la que llamaremos "la reunión." El evento lo organizó la comisión y fueron 
invitados los maestros de primaria que serían los tutores de los estudiantes de $7^{\circ}$ y $8^{\circ}$ semestres en las prácticas del preservicio.

La reunión se realizó en mayo de 2000, a tres meses del mes de agosto cuando se iniciarían las prácticas del preservicio. El lugar fue la sala audiovisual de la normal, espacio reservado para reuniones formales y para más de cien personas. Asistieron 90 maestros de primaria que fueron seleccionados (formalmente) como tutores por la Dirección de Educación Primaria del Estado. Algunos fueron capacitados por el Plan de Actividades Culturales de Apoyo a la Educación Primaria (PACAEP)

${ }^{8}$. En la reunión también se encontraban algunos directores y supervisores de escuelas primarias, así como el coordinador del PACAEP en el estado. Estaban los miembros de la comisión, así como profesores del Centro de Investigaciones de la normal y maestros de la Licenciatura en Educación Primaria.

Presidieron la reunión el Director General de la Normal, el Director del Departamento de Educación Primaria en el estado, la representante de la Secretaría de Educación Pública en el Estado y el Coordinador de la Licenciatura en Educación Primaria de la Normal. Como invitados estaban tres directores de escuelas normales particulares, así como un alumno y una alumna del $7^{\circ}$ semestre de la Licenciatura en Educación Primaria quienes dieron la bienvenida a los docentes que fungirían como sus tutores.

Las autoridades presentes en la reunión, además de conferir oficialidad a ésta, explicaron la existencia de convenios ya acordados entre las diferentes instancias educativas internas y externas a la normal involucradas en las prácticas del preservicio.

En la primera parte de la reunión, el director de la normal y el de Educación Primaria en el Estado, hablaron sobre la importancia de las prácticas escolares en el nuevo plan para la formación inicial de docentes. Asimismo, señalaron de manera muy general las actividades que deberían realizar los tutores durante las prácticas de los estudiantes. También presentaron a los tutores el calendario de las prácticas escolares que llegó a la normal desde la Secretaría de Educación Pública (SEP).

El director de la normal anunció que "un equipo de docentes" (refiriéndose a la comisión) se encontraba "elaborando un proyecto para el desarrollo de las prácticas escolares" y que ese equipo les explicaría la orientación de las tareas de los estudiantes, de los asesores y de los tutores durante las prácticas. El director también expresó, que los estudiantes que iniciarían las prácticas, habían llevado de manera extracurricular, cursos y talleres del PACAEP $^{9}$. Explicó además que una parte de los profesores de primaria que serían tutores de los estudiantes se habían capacitado en el PACAEP y que otra parte, como el $40 \%$ de ellos, pertenecían a escuelas multigrado ${ }^{10}$
El director agregó que los estudiantes del $7^{\circ}$ semestre asistirían a las primarias durante quince días para elaborar un proyecto que les permitiría cumplir con el servicio social. Señaló igualmente que el proyecto del ss que deberían preparar los estudiantes antes de iniciar el ciclo escolar, se desarrollaría como parte de las prácticas.

Se trataba, dijo el director, de un proyecto comunitario que partiría de los planteamientos del PACAEP y que orientaría la intervención de los estudiantes en las aulas, en la escuela y en la comunidad. Esta perspectiva para el desarrollo de las prácticas permitía a los estudiantes de la normal cumplir con sus prácticas a la vez que con su servicio social, según lo señalaron el director y el coordinador de la licenciatura.

En la segunda parte de la reunión la maestra Mariana, asignada por la comisión para ello, presentó la propuesta para las actividades académicas durante las prácticas, en la cual precisaba la forma en que los estudiantes organizarían el trabajo en las primarias acorde con el modelo de PACAEP. La maestra explicó a los tutores, que los estudiantes, durante sus prácticas, debían organizar el trabajo académico en las aulas a través de un proyecto escolar comunitario. Y, a manera de sugerencia, solicitó que los tutores se incorporaran a esos proyectos comunitarios. Después agregó que se trataba de impulsar a los estudiantes a desarrollar un trabajo alternativo, "diferente" al que tradicionalmente se les había enseñado en la normal, e involucrar en ese trabajo a toda la escuela primaria y a la comunidad.

Mariana: Para el proyecto escolar, la idea es una cooperativa, que los maestros se integren en un proyecto común para incidir en el ámbito comunitario... para ayudar no sólo a los practicantes a trabajar de un modo alternativo con sus alumnos, sino a toda la escuela, que sea una auténtica comunidad guiada en torno a proyectos educativos comunes (...) La idea es hacer equipos básicos; ustedes [los tutores de primaria], los practicantes y nosotros los asesores de la normal, para tener un proyecto común y promover proyectos de desarrollo comunitario y generar en la sociedad una nueva percepción del trabajo docente (...) Después ya sería la fase de las prácticas, cuando los muchachos ya estén practicando con el proyecto del PACAEP a lo largo de todo el año, como lo hacen los maestros de actividades culturales. Proponemos que los estudiantes trabajen con el sistema de proyectos durante todo el tiempo en que estén con el grupo, pero en trabajo cooperativo (...) y que ustedes [los tutores] permitieran a los estudiantes trabajar siguiendo los lineamientos de PACAEP" [aunque Uds. no hayan participado en ese programa]" V1/RT/11-05-00.

La propuesta que expuso la maestra Mariana en la reunión implicaba un cierto tipo de acciones para las 
prácticas en las primarias. Ellas incluían las formas de relación de los estudiantes con los tutores, con los alumnos de primaria, los padres de familia y, de manera más amplia, con la población en donde se ubicaba cada primaria de prácticas.

Este modelo estaba basado en la orientación pedagógica del PACAEP, misma que proponía la elaboración y el desarrollo de un proyecto comunitario. La propuesta, acordada entre la comisión, las autoridades educativas del estado y de la normal, era la expresión de un proceso de micropolítica frente al P97 en cuanto a la selección de tutores y de las tareas que se estaban asignando a los estudiantes para las prácticas.

Es decir, las tareas que fueron planteadas para los estudiantes en la reunión, eran distintas a las del P97. Algunas de las diferencias consistían en que el P97 establecía que las prácticas en el aula deberían planificarse por los estudiantes en concordancia con las actividades que en ellas desarrollaban los tutores, mismas que debían basarse en el plan de estudios de primaria de 1993 (SEP, 2000a, p. 13-16; SEP, 2000b, p. 12).

Asimismo, el P97 especificaba que las funciones de los profesores tutores eran: "orientar y asesorar al estudiante, hacer recomendaciones para las formas de trabajo, sugerir las estrategias y recursos en el aula y valorar el trabajo del estudiante" (SEP, 2000a, SEP, 2002, p. 19). En el mismo documento se decía que los estudiantes normalistas debían ser conducidos al trabajo docente de manera paulatina y bajo la ayuda del tutor; igualmente, entre los dos (estudiante y tutor), elaborarían y desarrollarían el plan de trabajo. De esas actividades debía tener conocimiento y participación el maestro asesor de la normal (SEP, 2002, p. 19-20).

Sin embargo, la propuesta que se presentó en la reunión daba prioridad y autonomía a los estudiantes para que planificaran y desarrollaran las actividades en las aulas bajo la orientación de un proyecto comunitario al que se adscribirían los tutores, dejando a éstos en un segundo plano. Se decidió esto a pesar de que se indicaba, en un documento de la SEP sobre el P97, que "los profesores de educación primaria, como expertos, cumplirán la función de asesoría durante las observaciones y prácticas educativas en las aulas, guiando a los estudiantes en los procedimientos y toma de decisiones, trasmitiendo sus saberes y experiencias en el trabajo con grupos escolares" (SEP, 2002, p. 19).

Otra cuestión donde contrastan las especificaciones del P97 para las prácticas de los estudiantes con lo que se presentó en la reunión, se refiere a los criterios para seleccionar a los tutores. Según las recomendaciones del P97, señalaban que éstos deberían elegirse de acuerdo con su desempeño académico, siguiendo las sugerencias de los supervisores de zona y directores de las escuelas primarias; en cambio, en la reunión se mostraba que en dicha selección se dio prioridad a los profesores habilitados por el PACAEP.

En la reunión, las autoridades educativas estatales, así como el director de la normal estaban de acuerdo con la propuesta de la comisión que presentó la maestra Mariana. Es decir, accedieron a que la propuesta del P97 fuera modificada en varios aspectos básicos, tales como la forma en que los estudiantes de la normal iban a organizar las actividades académicas en las primarias, así como en los criterios para la selección de los tutores.

En ese sentido consideramos, retomando el modelo explicativo de Wenger (1998), que en la normal del estudio tuvo lugar un proceso de negociación de significado entre todo el personal involucrado y el P97 oficial, el cual representaba la norma nacional. Esa negociación desencadenó el desarrollo de las micropolíticas a través de las cuales, los protagonistas incidieron para que sus ideas y perspectivas se objetivaran en acciones concretas, mismas que eran diferentes (en algunos aspectos) a las que se proponían en el P97. De esa manera, las micropolíticas emprendidas por los docentes y directivos de la normal se transformaron en políticas institucionales al ser aceptadas y promovidas por las diferentes autoridades educativas estatales.

Los procesos de micropolítica aquí descritos que protagonizaron los docentes, directivos y autoridades educativas estatales sobre el servicio social y el enfoque pedagógico para las prácticas fueron impulsados por la comunidad de práctica cultivada en la normal. En dichos procesos incidieron las experiencias previas de los docentes en la formación inicial, con el anterior plan de estudios, con las prácticas en las primarias, con el PACAEP, así como sus creencias y expectativas acerca de los estudiantes y los tutores.

\section{A Manera de Conclusiones}

El estudio nos permitió explicar, desde una perspectiva sociocultural, la manera en que los docentes y directivos de la normal del estudio se organizaron, a pesar de las confrontaciones internas entre ellos, construyendo una perspectiva local que les permitió desarrollar procesos de negociación de significado y micropolítica frente a la propuesta nacional del P97.

Los docentes y directivos se involucraron en una empresa común para superar los retos que les implicó la puesta en operación de las prácticas del preservicio con el P97. A partir de ello idearon y construyeron, colectivamente, estrategias así como nuevas formas de organización que se concretaron en los trabajos de la comisión, la cual funcionó como una comunidad de práctica cultivada (Wenger, McDermott \& Snyder, 2002). Los profesores que la constituyeron tenían experiencia en la coordinación de las prácticas escolares, y 
conocimientos sobre esas actividades. La comisión funcionó con cierta autonomía para tomar determinaciones enfocadas a la organización de las prácticas del preservicio.

El personal docente de la normal mostró capacidad para resolver los problemas que en esos momentos les planteaba el preservicio de los estudiantes. De esa manera lograron crear generando incluso un marco de acción interinstitucional y normativo que facilitó a las licenciaturas de preescolar, educación física y educación media la organización del preservicio que se inició en ellas posteriormente.

El análisis aquí presentado permitió mostrar procesos específicos de negociación de significado y micropolítica en prácticas cotidianas concretas dentro de un contexto de reforma a la formación inicial.

Recurriendo a Viñao (1997) podemos decir, como mostramos en el estudio, que las reformas educativas son interpretadas y puestas en práctica a partir de las experiencias, tradiciones, creencias valores y normas que existen en las escuelas (mismas que se encuentran constantemente en recomposición). En esos procesos intervienen elementos culturales aunados a las situaciones particulares por las que pasan los centros educativos y los mismos profesores en cada momento de reforma.

En ese sentido, por más que las reformas con frecuencia sean preparadas e implementadas desde los centros de poder estatal y se presenten con carácter normativo nacional, no pueden imponerse, ni llegar a la práctica tal y como son diseñadas. Toda reforma ha de pasar, en el plano de lo cotidiano, por procesos de negociación de significado desde los maestros y demás involucrados en las culturas propias de las escuelas. Sabemos que es común afirmar que las reformas se transforman en el camino de llegar a las realidades escolares; sin embargo, hace falta más investigación para conocer cómo ocurre esto. Creemos que para ello puede resultar fructífera la perspectiva teórica y metodológica que aquí adoptamos.

Finalmente, nos parece necesario emprender investigaciones que nos ayuden a explicar los procesos cotidianos que acontecen en la formación inicial de docentes, fundamentalmente sobre las prácticas del preservicio, que nos den cuenta de las experiencias derivadas de los últimos cambios en la formación inicial de maestros.

\section{Notas}

${ }^{1}$ En el término "directivos" incluimos al personal que dirigía la normal: el director y subdirector, los coordinadores de las licenciaturas y de los departamentos, así como los responsables de las unidades externas.

${ }^{2}$ En México el plan de estudios para las normales es de carácter nacional y normativo y es emitido por la Secretaría de Educación Pública.
${ }^{3}$ Este Programa estuvo integrado por cuatro líneas: Desarrollo curricular, Apoyo y formación continua, Fortalecimiento institucional y Elaboración de normas y orientaciones (Sep 1997b).

${ }^{4}$ En 1997 se inició la reforma del Plan del Estudios de la Licenciatura en Educación Primaria y posteriormente en las de Educación Preescolar, Educación Media, Educación Física y Educación Especial.

${ }^{5}$ La Subsecretaría de Educación Básica y Normal de la SEP fue la instancia que elaboró los planes y programas de 1997 para la formación de maestros en el país.

${ }^{6}$ Durante la investigación registramos, con apoyo de audio y video, además de eventos cotidianos, diversas reuniones entre directivos, docentes de la normal y maestros tutores, así como de estudiantes con asesores o con otros estudiantes. Sumaron 52 eventos, de los cuales tomamos dos para este artículo.

${ }^{7}$ El SNTE representa laboralmente a la mayoría del magisterio del país ante la SEP. El sindicato de los profesores de la normal del estudio es de carácter local y no pertenece al SNTE.

${ }^{8} \mathrm{El}$ PACAEP fue un programa nacional que capacitaba a docentes bajo un enfoque cultural y artístico. Ellos asesoraban a maestros de primaria (SEP, 1998). A la fecha del estudio este Programa ya no estaba vigente, excepto en algunos lugares.

${ }^{9}$ Según dijo el coordinador estatal del PACAEP en la reunión, la normal del estudio fue la única en el país que adoptó este programa para las prácticas de los estudiantes con el P97.

${ }^{10}$ En México, a las escuelas donde los maestros atienden en un solo grupo a niños de diferentes grados se les denomina multigrado.

\section{Bibliografía}

Ball S. J. (1987). La micropolítica en la escuela. Hacia una teoría de la organización escolar. España: Paidós.

Blase, J. (1997). La micropolítica de la enseñanza. En B. J. Biddle, T. L. Good, e I. F. Goodson (Eds.), La enseñanza y los profesores, vol. 2, (pp. 253-289). España: Paidós.

Bullogh R. Jr. (1997). Convertirse en profesor: La persona y la localización social de la formación del profesorado. En B. J. Biddle, T. L. Good I. F. Goodson (Eds.), La enseñanza y los profesores, vol. 1, (pp. 99-165). España: Paidós.

Chainklin, S. \& Lave J. (1996). Understanding practice. USA: Cambridge University Press.

Cole, M. (1996). Psicología cultural. España: Morata.

Coll, C. (1990). Constructivismo y educación: La concepción constructivista de la enseñanza y el aprendizaje. En Coll C. Palacios y Marchesi (Eds.), Desarrollo psicológico y educación, vol. 2, (pp. 158-190). España: Alianza.

Czarny, G., Popoca C., Castañeda M y López J. (1999). Formas de trabajo docente y su relación con los rasgos del perfil de egreso. México: Secretaría de Educación Pública (SEP).

Duverger, M. (1972). The study of politics. Londres: Nelson.

Erickson, F. (1989). Métodos cualitativos de investigación sobre la enseñanza. En Witrock. M. (Ed) La investigación de la enseñanza.Vol. II,(195-294). España: Paidós.

Fullan, M. (2002). Los nuevos significados del cambio en la educación. España: Octaedro.

Gal, N. (2006). The role of practicum, supervisors in behavior management education. Teaching and Teacher Education, 22, 377-393.

Geertz, C. (1973). La interpretación de las culturas. México: Gedisa 
Hargreaves, A. (2001). Aprender a cambiar. La enseñanza más allá de las materias y los niveles. España: Octaedro.

Hoyle, E. (1982). Micropolitics of educational organization. Educational Management and Administration. 10, 87-98.

Jay, J. K. \& Johnson, K. L. (2002). Capturing complexity: a typology of reflective practice for teacher education. Teaching and Teacher Education, 18, 73-85.

Kelchtermans, G. \& Katrijn, B. (2002). The micropolitics of teacher induction. A narrative biographical study on teacher socialization. Teaching and Teacher Education, 18, 105-120.

Lave, J. \& Wenger, E. (1991). Situated learning; legitimate peripheral participation. USA: Cambridge University Press.

Mercado, R. (1994). Formar para la docencia: Un reto de la educación normal, Universidad Futura, invierno, vol. 6, n. 16, 27-37.

Mercado, R. (2000). La implantación del plan 1997 de la licenciatura en educación primaria. México: SEP.

Mercado, R. (2002). Los saberes docentes como construcción social. México: Fondo de Cultura Económica.

Putnam, R. y Borko, H. (1997). El aprendizaje del profesor: implicaciones de las nuevas perspectivas de la cognición. En B. J. Biddle, T. L. Good e I. F. Goodson (Eds.), La enseñanza y los profesores, vol. 1, (pp. 219-329). España: Paidós.

Rockwell, E. (1980). Etnografía y teoría en la investigación educativa. México: Departamento de Investigaciones Educativas (DIE) CINVESTAV-IPN.

Rockwell, E. y Mercado, R. (1986). La práctica docente y la formación de maestros. En Rockwell E. y Mercado R. (Eds.). La escuela lugar del trabajo docente, (pp. 63-78). México: DIE-CINVESTAV-IPN.

Rogoff, B. (1990). Aprendices del pensamiento. España: Paidós.

Salomon, G. (1993). Cogniciones distribuidas. Consideraciones psicológicas y educativas. Argentina: Amorrortu.

Schön, D. (1987). La formación de profesionales reflexivos. Hacia un nuevo diseño de la enseñanza y el aprendizaje en las profesiones. España: Paidós.

SEP (1997a). Plan de estudios de la licenciatura en educación primaria. México: SEP.

SEP (1997b). Programa para la transformación y el fortalecimiento académico de las escuelas normales. México: SEP.

SEP (1997c). XLV Conferencia internacional sobre educación. México: SEP.

SEP (1998). Programa de apoyo a la educación primaria. México: SEP.

SEP (2000a). Lineamientos para la organización del trabajo académico durante séptimo y octavo semestres de la licenciatura en educación primaria plan 1997. México: SEP.

SEP (2000b). Lineamientos para el desarrollo de las actividades de acercamiento a la práctica escolar y su relación con los cursos de las demás Asignaturas. México: SEP.

SEP (2002). Las actividades de observación y práctica en la escuela primaria. México: SEP.

Sim, C. (2005). Preparing for professional experiences incorporating pre-service teachers as communities of practice. Teaching and Teacher Education, 22, 77-83.

Taitel, L. (1997). Professional development schools and the transformation of teacher leadership. Teacher Education Quarterly, winter, 9-22.

Veenman, S., Benthum N., Bootsma D., Dieren J. \& Kemp N. (2002). Cooperative learning and teacher education. Teaching and Teacher Education, 18, 87-103.

Vygotski, L. (1978). El desarrollo de los procesos psicológicos superiores. España: Editorial Crítica.

Viñao, A. (1997). Las instituciones y culturas escolares en su pers- pectiva socio-histórica: tradiciones y cambios. España: Editorial ICE.

Viñao, A. (2001). La cultura de las reformas escolares. La perspectiva docente, 26, 38-56, segunda época.

Wenger, E. (1998). Comunidades de práctica. Aprendizaje significativo e identidad. España: Paidós.

Wenger, E., McDermott, R. \& Snyder, W. (2002). Cultivating communities of practice. USA: Harvard Business School Press.

Wilson, E. (2006). The impact of an alternative model of student teacher supervision: Views of the participants. Teaching and Teacher Education, 22, 22-31.

Woods, P. (1986). La escuela por dentro. La etnografía en la investigación educativa. España: Paidós.

Wubbels, T. Kortagen, F. \& Brekelmans, M. (1997). Developing theory from practice in teacher education. Teacher Education Quarterly, summer, 75-90.

Pedro Antonio Estrada Rodríguez és licenciado em Filosofia pela Universidad Michoacana de San Nicolás de Hidalgo, Graduado de Maestría en Ciencias de

la Educación en la Universidad Autônoma de Querétaro, Doutorando em Ciencias en la Especialidad de Investigaciones Educativas en el Centro de Investigación y de Estudios Avanzados (CINVESTAV) del Instituto Politécnico Nacional. Professor na Escuela Normal del Estado de Querétaro y Asesor en la Universidad Pedagógica Nacional. México D.F., México. Endereço para correspondência: Veracruz, 216, Colonia San José el Alto, C.P. 76139. Querétaro, Qro. México. Email: pedroan52@yahoo.com.mx

Ruth Mercado Maldonado és graduada de Maestría en Ciencias em la Especialidad de Investigaciones Educativas, por el Centro de Investigación y de

Estudios Avanzados del Instituto Politécnico Nacional, Doutora em Ciencias em la Especialidad de Investigaciones Educativas del DIE. Investigadora en el DIE del CINVESTAV e docente nos programas de maestría y doctorado en el DIE del CINVESTAV. Endereço para correspondência: Calzada de los tenorios 235 Colonia Granjas Coapa. México DF. Código Postal 14330. México.

Email: $\underline{\text { rmercado@ cinvestav.mx }}$

\author{
Procesos de negociación de significado \\ en una escuela normal mexicana \\ Pedro Antonio Estrada Rodríguez \\ e Ruth Mercado Maldonado
}

Recebido: $26 / 08 / 2008$

Aceite final: 15/12/2008 